Altenpflege

\title{
Suchtkrank im Alter
}

— Wie verhalte ich mich als Pflegekraft, wenn ich den Eindruck habe, dass ein Bewohner in meiner Altenpflegeeinrichtung ein Suchtproblem hat? Nur in wenigen Einrichtungen gibt es Konzepte zum Umgang damit. Wissenschaftler der Katholischen Hochschule Nordrhein-Westfalen (KatHO NRW) haben nun Handlungsempfehlungen erarbeitet.

„Es ist oft nicht leicht, bei älteren Personen eine Suchtproblematik zu erkennen", sagt Projektleiterin Prof. Dr. Tanja Hoff, "da bei ihnen zum Beispiel Trinkexzesse oder impulsive, aggressive Äußerungen seltener vorkommen. Darüber hinaus ist es oft schwierig, zwischen altersbedingten Veränderungen und Folgen von Suchtmittelkonsum zu unterscheiden. Ein Beispiel sind zittrige Hände." Für die Bewohner sei es aber zum Teil lebenswichtig, dass die Pflegekräfte Entzugserscheinungen oder eine mögliche Vergif-

tung rechtzeitig erkennen und richtig reagieren. Die Handlungsempfehlungen enthalten Vordrucke, Frage- und Dokumentationsbögen sowie Checklisten, die den Pflegekräften den Umgang mit suchtkranken oder -gefährdeten Bewohnern erleichtern sollen. Gefördert wurde das Forschungsprojekt „Sucht im Alter - Netz- und netzwerkbasierte Optimierung der ambulanten und stationären Pflege" (SANOPSA) vom Bundesforschungsministerium im Rahmen der Förderlinie "SILQUA-FH“ des Programms „Forschung an Fachhochschulen".

www.sanopsa.de

\section{Pflegeunternehmen organisieren Kinderbetreuung Erfolgsfaktor Familie}

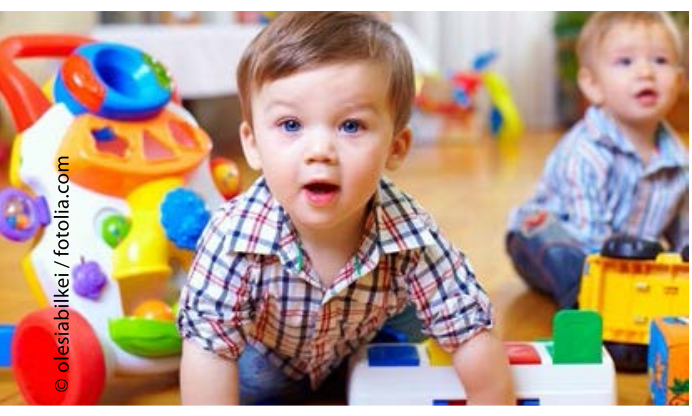

— Um ihre Fachkräfte zu halten und im Arbeitsalltag zu entlasten, bieten private Pflegedienste und Heime verstärkt Unterstützung bei der Kinderbetreuung. In einer von den Wirtschaftsförderungsgesellschaften der Länder Berlin und Brandenburg in Auftrag gegebenen „Einrichtungsbefragung zur Situation in ausgewählten Gesundheitsfachberufen" gaben 36\% der Pflegeunternehmen in Brandenburg an, ihre Mitarbeiterinnen und Mitarbeiter in diesem Bereich zu entlasten - deutlich mehr als in Berlin, dort sind es $26 \%$. Außerdem bewerteten die befragten
Einrichtungen diese Form der Unterstützung besonders hoch.

„Die Arbeit in der Pflege erfordert viel zeitliche Flexibilität, deshalb wissen es die Fachkräfte zu schätzen, wenn sich die Unternehmen auch Gedanken darüber machen, wie Arbeitsalltag und Kinderbetreuung koordiniert werden können", erklärt die brandenburgische Landesvorsitzende des Bundesverbandes privater Anbieter sozialer Dienste e.V. (bpa) Ellen Fährmann.

Auch eine Umfrage des bpa in Zusammenarbeit mit dem Projekt "Erfolgsfaktor Familie" habe ergeben, dass die Kinderbetreuung einen wichtigen Baustein für eine mitarbeiterfreundliche Unternehmenskultur darstelle, so Fährmann. „Den Mitarbeiterinnen und Mitarbeitern ist es wichtig, dass sie Job und Privatleben miteinander in Einklang bringen können. Das schließt auch eine staltung ein und kann helfen, Fachkräfte zu gewinnen und zu halten."

www.bpa.de gewisse Flexibilität bei der Dienstplange-

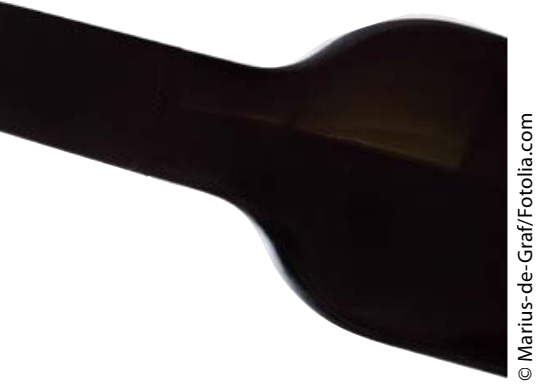

Gesundheit fördern in der Pflege

— Konzepte und Methoden der Gesundheitsförderung und Prävention müssen sich verstärkt an Pflegekräfte selbst wenden, die täglich mit Stress, körperlichen Beschwerden wie Rückenschmerzen und Überlastungen zu kämpfen haben. Der ELearning-Kurs „Fachkraft für Gesundheitsförderung in der Pflege" vermittelt kompaktes und gesundheitsförderliches Wissen, das zur Planung und Umsetzung gesundheitsförderlicher und präventiver Maßnahmen und Projekte in ihrem Praxisfeld genutzt werden und zur Entlastung des Pflegeteams beitragen kann. Des Weiteren werden Kompetenzen zur Verbesserung der Kommunikation im Pflegeprozess, in der Teamkommunikation und Konfliktlösung diskutiert. Methoden der Stressprävention und des Zeitmanagements komplettieren das Kursangebot und können im Bereich der Verhaltens- und Verhältnisprävention und zur Stärkung teamspezifischer Ressourcen genutzt werden. Der Kurs richtet sich an Kranken- und Gesundheitspfleger, Altenpfleger sowie Absolventen eines Bachelor-Studiums im Bereich der Pflegewissenschaft. Der Beginn des E-Learning-Kurses, der von der Ländliche Erwachsenenbildung in Niedersachsen e.V., Fachbereich Gesundheit und Selbsthilfe, angeboten wird, ist für das 4. Quartal 2015 vorgesehen.

www.bildungspraemie.info 